\title{
Increased expression of microRNA-503 and reduced expression of kangai-1 in B-cell non-Hodgkin's lymphoma
}

\author{
JINGJING WU* ${ }^{*}$ AIMIN LI* , PENGYU ZHANG, ZHENCHANG SUN, LIJUAN HAN, FEIFEI NAN and LI GENG \\ Department of Oncology, Institute of Clinical Medicine, The First Affiliated Hospital of Zhengzhou University, \\ Zhengzhou, Henan 450052, P.R. China
}

Received September 29, 2014; Accepted August 5, 2015

DOI: $10.3892 /$ etm.2016.2971

\begin{abstract}
The aim of the present study was to determine the expression levels of microRNA-503 (miR-503) and the tumor suppressor gene, kangai-1 (KAI1), in B-cell non-Hodgkin's lymphoma (B-NHL). A total of 45 patients with B-NHL (including 29 cases with stage III/IV disease and 16 cases with stage I/II disease) were enrolled in this study. In addition, 26 patients with reactive lymphoid hyperplasia (RLH) were enrolled as the control patients. Reverse transcription-quantitative polymerase chain reaction was performed in order to measure the expression levels of miR-503 in B-NHL and RLH tissues, and to detect the expression levels of miR-503 and KAI1 in peripheral blood samples. In addition, KAI1 expression levels in B-NHL and RLH tissues were detected using western blotting and immunohistochemical analysis. The expression levels of miR-503 were found to be significantly increased in the tissues and peripheral blood of B-NHL patients when compared with those in RLH patients $(\mathrm{P}<0.05)$. However, KAI1 was strongly expressed in RLH tissues and weakly expressed in B-NHL tissues. Furthermore, the expression levels of KAI1 were significantly decreased in the tissues and peripheral blood of B-NHL patients when compared with those in the tissues and peripheral blood of RLH patients $(\mathrm{P}<0.05)$. The expression levels of miR-503 in the tissues and peripheral blood of patients with stage III/IV B-NHL were significantly higher compared with those with stage I/II B-NHL $(\mathrm{P}<0.05)$. By contrast, the expression levels of KAI1 in stage III/IV B-NHL tissues were significantly higher compared with those in stage I/II B-NHL tissues $(\mathrm{P}<0.05)$. In conclusion, miR-503 was highly expressed, whereas KAI1 was poorly expressed,
\end{abstract}

Correspondence to: Dr Li Geng, Department of Oncology, Institute of Clinical Medicine, The First Affiliated Hospital of Zhengzhou University, 1 Jianshe East Road, Zhengzhou, Henan 450052, P.R. China

E-mail: zhangmz03@163.com

*Contributed equally

Key words: B-cell non-Hodgkin's lymphoma, microRNA-503, kangai-1 in the tissues and peripheral blood of B-NHL patients. Thus, miR-503 may have an application as a novel therapeutic and diagnostic marker in B-NHL patients.

\section{Introduction}

MicroRNA (miRNA or miR) is a small endogenic non-coding RNA molecule that regulates gene expression by binding to 3'-untranslated regions of target mRNA (1). The expression profile of miRNA is significantly altered in tumor tissues, which suggests that miRNA molecules may play an important role in the development of tumors. Therefore, miRNA molecules have great application prospect in the clinical treatment and diagnosis of tumors $(2,3)$.

miR-503 is a recently-discovered tumor miRNA molecule $(4,5)$ The expression levels of miR-503 are associated with various tumor tissue types (6) and have been found to be reduced in oral cancer (7), hepatocellular carcinoma (8) and endometrial carcinoma (6), when compared with the levels in normal tissues. Xiao et al (9) found that low expression levels of miR-503 were associated with tumor node metastasis staging of hepatocellular carcinoma (10). However, in adrenal carcinoma, parathyroid carcinoma and retinoblastoma, the expression levels of miR-503 are higher compared with those in normal tissues (11). Kangai-1 (KAI1) is a common tumor suppressor gene that is associated with the invasion, migration and prognosis of numerous tumors, including laryngeal squamous cell carcinoma and prostate, lung, liver and breast cancer $(12,13)$.

B-cell non-Hodgkin's lymphoma (B-NHL) is a common cancer of the immune system, which mainly occurs in the lymph node tissues $(14,15)$. In China, B-NHL accounts for 61.6-74.2\% of all NHL cases (15). Due to the fast progression and high invasiveness of this disease, the prognosis of B-NHL patients is relatively poor (16). To the best of our knowledge, the expression levels of miR-503 and KAI1 have not been previously reported in B-NHL tissues. Through the analysis of the Kyoto Encyclopedia of Genes and Genomes (KEGG) database that was performed in the present study, it is hypothesized that KAI1 may be regulated by miR-503 in B-NHL tissues. Therefore, in the present study, the expression levels of miR-503 and KAI1 were detected in B-NHL tissues and the peripheral blood of patients with B-NHL. Subsequently, the association of the miR-503 and KAI1 expression levels with the occurrence and development of B-NHL was analyzed. 


\section{Materials and methods}

Reagents. The TRIzol reagent used for RNA extraction was purchased from Invitrogen (Thermo Fisher Scientific, Inc., Carlsbad, CA, USA). The PrimeScript RT Reagent kit and One Step SYBR PrimeScript RT-PCR kit II (Perfect Real Time) were purchased from Takara Bio Inc. (Tokyo, Japan). The rabbit anti-human KAI1 polyclonal antibody (cat no. ab66400; dilution 1:1,000), biotinylated goat anti-rabbit IgG (cat no., ab128978; dilution 1:5,000) rabbit anti-human anti- $\beta$-actin polyclonal antibody (cat no., 189073; dilution 1:5,000) and horseradish peroxidase (HRP)-conjugated goat anti-rabbit antibody (cat no. ab6721; dilution 1:5,000) were purchased from Abcam (Cambridge, MA, USA). Streptavidin-peroxidase immunohistochemical staining kits were purchased from Zhongshan Golden Bridge Biotechnology Co., Ltd. (Beijing, China). Radioimmunoprecipitation assay (RIPA) buffer and BeyoECL Plus enhanced chemiluminescence reagent were purchased from Beyotime Institute of Biotechnology (Haimen, China).

Clinical data. A total of 45 patients with B-NHL admitted to the First Affiliated Hospital of Zhengzhou University (Zhengzhou, China) between January 2013 and April 2014 were enrolled in the present study. There were no specific inclusion criteria in this study. These 45 patients with B-NHL included 23 males and 22 females, with an age range of 17-66 years and a mean age of 45.7 years. Among them, there were 29 cases with stage III/IV and 16 cases with stage I/II disease (Ann Arbor staging classification) (17). In addition, 26 patients with reactive lymphoid hyperplasia (RLH), including 12 males and 14 females with a mean age of 38 years, were included in this study as the control group. Lymphoma or healthy tissues were obtained from the B-NHL patients by surgery prior to treatment, and peripheral blood samples were collected from the cubital vein of the RHL patients prior to surgery. The diagnostic criteria for B-NHL or RHL were based on the 2008 revision of the World Health Organization classification of myeloid neoplasms and acute leukemia (18). B-NHL patients were treated with surgery and routine chemotherapy, while RHL patients were treated with surgery. Prior written informed consent was obtained from each patient. The study was approved by the Ethics Review Board of Zhengzhou University.

Immunohistochemical staining. All the tissue samples were rapidly frozen in liquid nitrogen. After fixing in formaldehyde and embedding in paraffin, the samples were cut into $4 \mu \mathrm{M}$ sections. Next, the sections were dewaxed and rehydrated in graded alcohols. In order to inactivate endogenous peroxidase, $3 \%$ freshly-prepared hydrogen peroxide was added and the sections were incubated at room temperature for $10 \mathrm{~min}$. Following antigen retrieval, rabbit anti-human KAI1 polyclonal antibody (dilution, 1:200) was added and the samples were incubated in the dark for $1 \mathrm{~h}$. After rinsing with phosphate-buffered saline, biotinylated goat anti-rabbit $\mathrm{IgG}$ antibody was added and incubated for $30 \mathrm{~min}$ at $37^{\circ} \mathrm{C}$. The sections were developed with $\mathrm{DAB}$ chromogenic reagent and counterstained with hematoxylin (Beyotime Institute of Biotechology, Haimen, China). Subsequent to hydrochloric acid differentiation and dimethylbenzene transparency (19), the tissue sections were mounted with neutral gum (Bioworld Technology Co., Ltd., St Louis Park, MN, USA).

The samples were observed under a microscope (BX53; Olympus Optical Co., Ltd., Tokyo, Japan) and cells exhibiting brown staining were defined as KAI1-positive. Five fields at high-magnification were randomly selected and positive cells were counted. A positive rate referred to the ratio of the positive cell number relative to the total cell number. A minimum of 200 cells were counted in each sample. Based on the percentage of positive staining, the immunohistochemical staining results were scored based on a scoring system developed by our group: Score $0,0 \%$ positive staining; score $1,1-25 \%$ positive staining; score $2,26-50 \%$ positive staining; and score $3,51-100 \%$ positive staining. The immunohistochemical staining results were also scored based on the staining intensity, as follows: score 0 , no staining; score 1, light yellow staining; score 2, brownish yellow staining; and score 3, brown staining. Subsequently, the degree of staining was calculated by combining the scores of the positive staining percentage and the staining intensity. The overall degree of staining was defined as follows: score $0-1$, negative staining; score 2-3, weakly positive staining; score $4-6$, moderately positive staining; and score $>6$, strongly positive staining.

Reverse transcription-quantitative polymerase chain reaction $(R T-q P C R)$ assay. Total RNA was extracted from the tissues and peripheral blood samples using TRIzol reagent. All RNAs were reverse-transcribed into cDNA using the PrimeScript RT Reagent kits and qPCR assay was conducted using One Step SYBR PrimeScript RT-PCR kit II (Perfect Real Time), according to the manufacturer's instructions. According to the miR-503 and KAI1 sequences obtained from the GenBank database (KAI1, U20770.1; miR-503, MI0003188), primer sets were designed using the online Pubmed primer blast and synthesized by Invitrogen (Thermo Fisher Scientific, Inc.). The primer sequences used were as follows: For miR-503, 5'-TAG CAGCGGGAACAGTTC-3' (forward) and universal primer [reverse; One Step SYBR PrimeScript RT-PCR kit II (Perfect Real Time)]; for KAI1, 5'-GTTCTCTTATCAACTCAG-3' (forward) and 5'-TACAATATACACACCCTT-3' (reverse); for $\beta$-actin, 5'-CTCCATCCTGGCCTCGCTGT-3' (forward) and 5 -GCTGTCACCTTCACCGTTCC-3' (reverse). $\beta$-actin was used as an internal control. qPCR was performed on the ABI One-Step Plus thermal cycler (Applied Biosystems; Thermo Fisher Scientific, Inc., Foster City, CA, USA). The PCR system included $10 \mathrm{ul}$ qRT-PCR-Mix, $0.5 \mathrm{ul}$ forward primer, $0.5 \mathrm{ul}$ reverse primer, $1 \mathrm{ul} \mathrm{cDNA}$ and $8 \mathrm{ul} \mathrm{dd}_{2} \mathrm{O}$. The PCR cycle conditions for miR503 were as follows: Pre-denaturation at $95^{\circ} \mathrm{C}$ for $1 \mathrm{~min}$, and then 40 cycles of $95^{\circ} \mathrm{C}$ for $1 \mathrm{~min}$ and $60^{\circ} \mathrm{C}$ for $30 \mathrm{sec}$. The PCR cycle conditions for KAI1 were as follows: Pre-denaturation at $95^{\circ} \mathrm{C}$ for $5 \mathrm{~min}$ and then 40 cycles of $95^{\circ} \mathrm{C}$ for $1 \mathrm{~min}, 58^{\circ} \mathrm{C}$ for $30 \mathrm{sec}$ and $72^{\circ} \mathrm{C}$ for $30 \mathrm{sec}$. For each sample, the PCR reaction was repeated at least 3 times. The $2^{-\Delta \Delta \mathrm{Ct}}$ method was used to calculate the relative expression levels of miR-503 and KAI1.

Western blotting. Western blotting was performed as previously described (20). Briefly, tissues were homogenized in RIPA buffer. Total proteins were separated by $12 \%$ SDS-PAGE and then analyzed by immunoblotting. $\beta$-actin was used as 

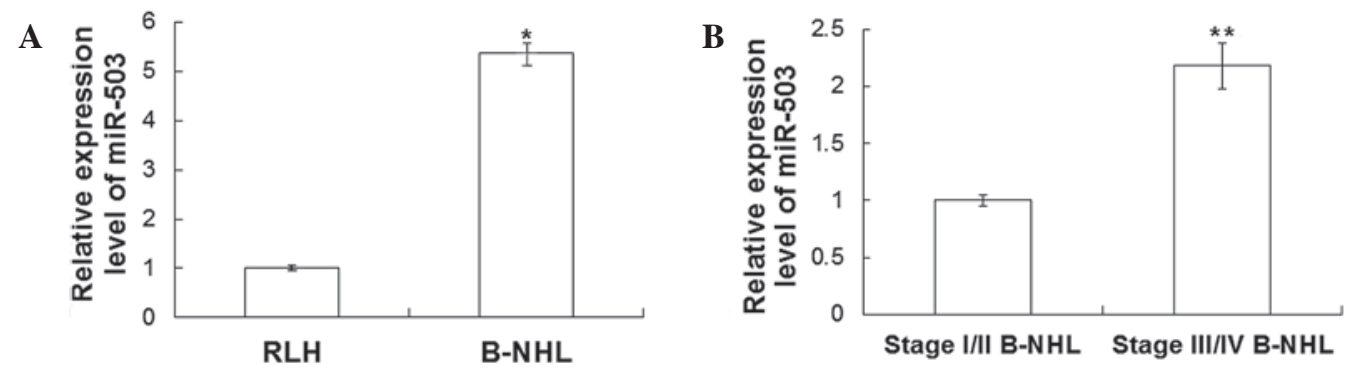

Figure 1. Histograms of normalized expression levels of miR-503 in (A) B-NHL and RLH tissues, and (B) stage I/II and stage III/IV B-NHL tissues. The expression levels of miR-503 were detected by reverse transcription-quantitative polymerase chain reaction assay. Experiments were repeated at least 3 times. Error bars indicate the standard error of the mean. "P<0.05, vs. RHL; "* P $<0.05$, vs. stage II B-NHL. miR, microRNA; B-NHL, B-cell non-Hodgkin's lymphoma; RHL, reactive lymphoid hyperplasia.

A

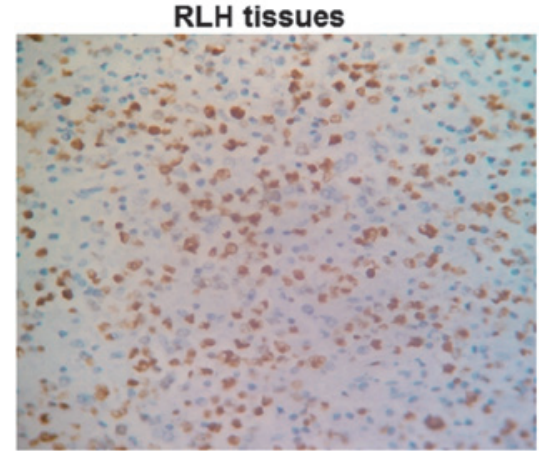

B

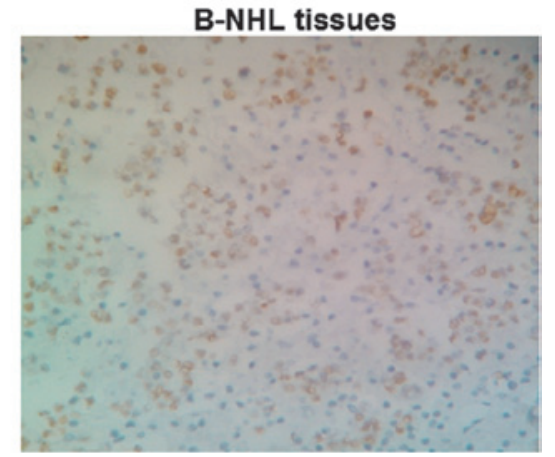

Figure 2. Representative immunohistochemical staining showing the expression of KAI1 in (A) RLH and (B) B-NHL tissues. Fields of sections were visualized using a high-resolution optical microscope at a magnification of x200. Cells with brown staining were defined as KAI1-positive. (A) Strongly positive expression of KAI1 was observed in RLH tissues, while (B) weakly positive expression of KAI1 was observed in B-NHL tissues. KAI1, kangai-1; B-NHL, B-cell non-Hodgkin's lymphoma; RHL, reactive lymphoid hyperplasia.

an internal control. The primary antibodies used in western blotting were the rabbit anti-human KAI1 polyclonal antibody (dilution, 1:500) and rabbit anti-human anti- $\beta$-actin polyclonal antibody (dilution, 1:5,000). The secondary antibody used in the present study was the HRP-conjugated goat anti-rabbit antibody (dilution, 1:2,000). Detection of the blots was performed using BeyoECL Plus enhanced chemiluminescence reagent and the blots were quantified using Quantity One software version 4.62 (Bio-Rad Laboratories, Inc., Hercules, CA, USA). The experiments were repeated at least 3 times.

Predicting the possible target genes of miR-503. The target genes of miR-503 were predicted using TargetScan version 7.0 (http://www.targetscan.org/). The signaling pathways that were found to be associated with the target genes of miR-503 were then analyzed using the KEGG database (http://www.genome.jp/kegg/). KAI1 was identified as one of the target genes of miR-503. The prediction using TargetScan and the analysis using KEGG were performed according to standard procedures (21).

Statistical analysis. All the results are expressed as the mean \pm standard deviation and statistical analyss were performed using SPSS version 16.0 for Windows (SPSS Inc., Chicago, IL, USA). Paired t-test was used to analyze comparisons between groups and for the analysis of paired data. $\chi^{2}$ test was used to analyze the relationship of KAI1 positive expression with the clinical staging of B-NHL.
$\mathrm{P}<0.05$ was considered to indicate a statistically significant difference.

\section{Results}

Expression levels of miR-503 are increased in B-NHL tissues. In order to detect the expression levels of miR-503 in B-NHL and RLH tissues, RT-qPCR assay was performed and the results are demonstrated in Fig. 1. As shown in Fig. 1A, the expression levels of miR-503 in B-NHL tissues were $\sim 5.36 \pm 0.21$-fold higher compared with those in RLH tissues $(\mathrm{P}<0.05)$. As shown in Fig. 1B, the miR-503 expression levels in B-NHL tissues of patients with stage III/IV disease were $\sim 2.18 \pm 0.20$-fold higher compared with those in patients with stage I/II disease $(\mathrm{P}<0.05$; Fig. 1B). These results indicated that miR-503 was highly expressed in B-NHL tissues and that its expression was higher in stage III/IV B-NHL tissues.

Expression levels of KAII are decreased in B-NHL tissues. To determine the expression of KAI1 protein in B-NHL and RLH tissues, immunohistochemical staining was conducted. Representative immunohistochemical staining results are shown in Fig. 2. Cells with brown staining were considered to be KAI1-positive. In RLH tissues, there were 7 cases with weakly positive expression of KAI1 (26.9\%) and 19 cases with strongly positive expression of KAI1 (73.1\%). A representative image of strongly positive KAI1 expression in RLH tissues is shown in Fig. 2A. However, in B-NHL tissues, there were 
A

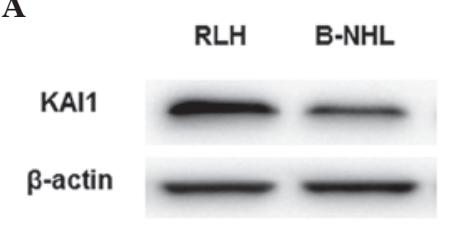

B

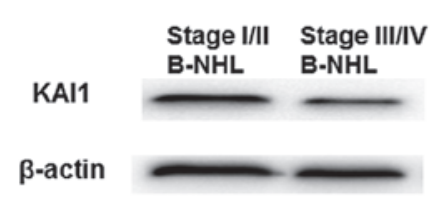

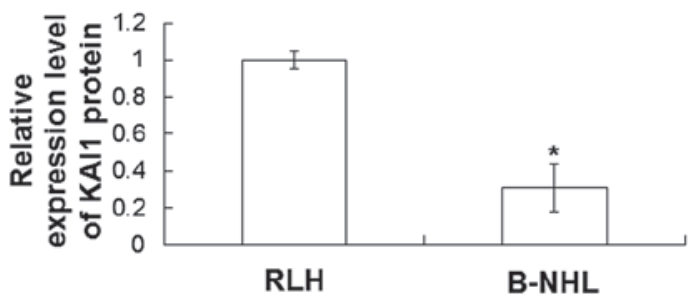

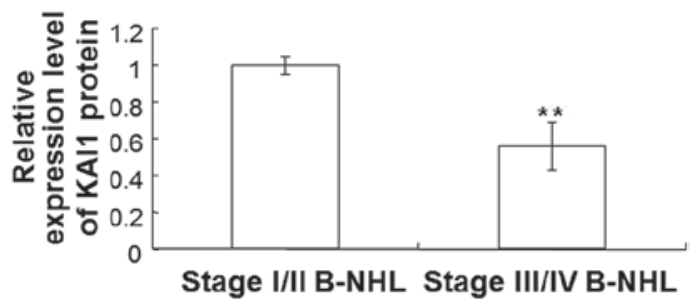

Figure 3. Expression levels of KAI1 in (A) B-NHL and RLH tissues, and (B) in B-NHL tissues of patients with stage I/II and III/IV disease, as detected by western blotting. Total proteins of B-NHL and RLH tissues were harvested and separated using 12\% SDS/PAGE, and then analyzed by western blotting. $\beta$-actin was used as a loading control. Left panels, representative western blotting results; right panels: quantitative western blotting results. ${ }^{*} \mathrm{P}<0.05$ vs. $\mathrm{RLH}$; ${ }^{* *} \mathrm{P}<0.05$, vs. stage I/II B-NHL. KAI1, kangai-1; B-NHL, B-cell non-Hodgkin's lymphoma; RHL, reactive lymphoid hyperplasia.
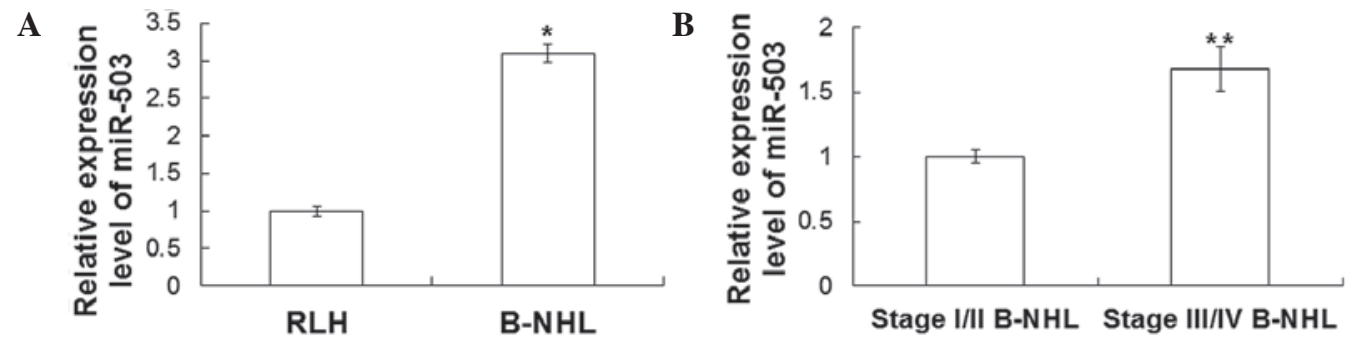

C

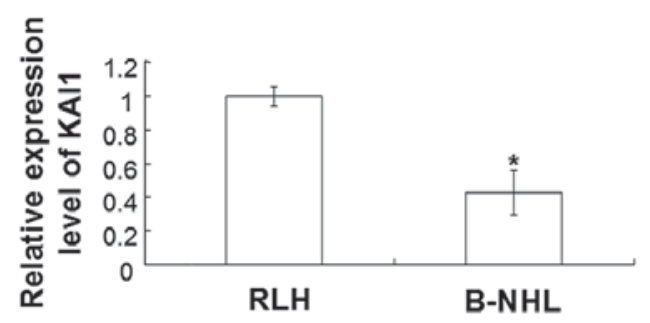

Figure 4. Histograms of the mean normalized expression levels of miR-503 in the peripheral blood of (A) RLH and B-NHL patients, and (B) B-NHL patients with stage I/II and stage III/IV disease. (C) Mean normalized expression levels of KAI1 in the peripheral blood of B-HNL and RLH patients. Reverse transcription-quantitative polymerase chain reaction assay was performed to detect the expression levels of miR-503 and KAI1 in the peripheral blood. Experiments were repeated at least 3 times. Error bars show standard error of the mean. " $\mathrm{P}<0.05$ vs. RLH; ${ }^{* *} \mathrm{P}<0.05$, vs. stage I/II B-NHL. KAI1, kangai-1; miR, microRNA; B-NHL, B-cell non-Hodgkin's lymphoma; RHL, reactive lymphoid hyperplasia.

37 cases with weakly positive KAI1 expression $(82.2 \%)$ and 8 cases with strongly positive KAI1 expression (17.8\%). A representative image of weakly positive KAI1 expression in B-NHL tissues is shown in Fig. 2B. There were no statistically significant differences in KAI1 expression among different ages and genders $(\mathrm{P}>0.05)$.

To further verify the expression of KAI1 protein, western blotting was performed in B-NHL and RLH tissues. As shown in Fig. 3, the expression of KAI1 protein was significantly decreased in B-NHL tissues when compared with that in RLH tissues $(\mathrm{P}<0.05)$. The expression levels of KAI1 in B-NHL tissues of patients with stage I/II and stage III/IV disease were also detected by western blotting. As shown in Fig. 3B, KAI1 expression in stage III/IV B-NHL tissues was significantly lower compared with that in stage I/II B-NHL tissues $(\mathrm{P}<0.05)$. These results indicate that the expression levels of KAI1 are decreased in B-NHL tissues and that KAI1 expression is lower in stage III/IV B-NHL tissues.

Expression levels of miR-503 are increased, whereas those of KAII $\mathrm{mRNA}$ are decreased in the peripheral blood. RT-qPCR was performed to detect the expression levels of miR-503 and KAI1 mRNA in peripheral blood. The RT-qPCR results are shown in Fig. 4. As shown in Fig. 4A, miR-503 expression levels in the peripheral blood of B-HNL patients were significantly increased when compared with those of RLH patients $(\mathrm{P}<0.05)$. In addition, miR-503 expression levels in the peripheral blood of patients with stage III/IV B-NHL were significantly increased when compared with those in patients with stage I/II B-NHL $(\mathrm{P}<0.05$; Fig. 4B). Furthermore, as shown in Fig. 4C, KAI1 expression levels were significantly decreased in the peripheral blood of B-NHL patients 
$(\mathrm{P}<0.05)$. However, no statistically significant differences in the KAI1 mRNA expression levels in the peripheral blood were detected between B-NHL patients with stage I/II and stage III/IV disease (data not shown). These results indicate that the expression levels of miR-503 and KAI1 in the peripheral blood of B-NHL patients are consistent with those in B-NHL tissues.

\section{Discussion}

Recently, the prognosis of B-NHL patients has improved significantly due to better treatments, including the application of radiotherapy, chemotherapy and immunotherapy; however, factors such as tumor cell metastasis, drug resistance and relapse affect the prognosis of patients with B-NHL (22). It has been that several factors, such as miRNA (23), CHFR prophase checkpoint gene (24) and human phosphatidylethanolaminebinding protein 4 (25), may be associated with B-NHL occurrence and development, the molecular mechanisms of which require further study.

Previous studies have reported that altered miRNA expression is associated with the development of lymphoma $(26,27)$. In the present study, immunohistochemical staining, western blotting and RT-qPCR assay were performed to investigate the expression levels of miR-503 and KAI1 in the tissues and peripheral blood of B-NHL patients. The results demonstrated that the expression levels of miR-503 were significantly increased in B-NHL tissues when compared with those in RLH tissues. In addition, the miR-503 expression levels in B-NHL tissues of patients with stage III/IV disease were increased when compared with those with stage I/II disease. The expression levels of miR-503 in the peripheral blood of B-NHL patients were detected using RT-qPCR, which revealed that the levels were significantly increased compared with those in the peripheral blood of RLH patients. Furthermore, miR-503 expression levels in the peripheral blood of patients with stage III/IV B-NHL were significantly increased when compared with those in stage I/II B-NHL patients. However, previous studies have indicated that miR-503 expression levels were downregulated in endometrioid endometrial cancer (6) and gastric cancer (28). This difference in the expression profile of miR-503 between our results and the results of previous studies may be caused by the difference in the cancer type. In addition, the expression levels of miR-503 in B-NHL tissues and peripheral blood changed with the pathological stage of B-NHL, which indicates that miR-503 may be used as a molecular marker for the prognosis of B-NHL.

Maurer et al (29) reported that KAI1 expression was decreased in advanced-stage colon cancer. In addition, Guo et al (30) identified that KAI1 expression was increased in early pancreatic cancer and decreased in the presence of metastases. However, Yang et al (31) reported that KAI1 expression was reduced in non-metastatic human colorectal cancer, but was regained in metastatic human colorectal cancer. Through the analysis of the KEGG database that was performed in the present study, we hypothesized that KAI1 may be regulated by miR-503 in B-NHL tissues. As revealed by immunohistochemical staining, KAI1 expression was weakly positive in B-NHL tissues and strongly positive in RHL tissues. Similarly, the expression levels of KAI1 protein were significantly decreased in B-NHL tissues. In addition, KAI1 expression levels in the tissues of B-NHL patients with stage III/IV disease were decreased when compared with those of patients with stage I/II disease. Furthermore, KAI1 mRNA expression levels were significantly decreased in the peripheral blood of B-NHL patients. However, no statistically significant differences in the KAI1 mRNA expression levels were detected between patients with stage I/II B-NHL and patients with stage III/IV B-NHL. These results were consistent with the findings of Maurer et al (29) and Guo et al (30), which indicated that the expression levels of KAI1 are decreased in B-NHL tissues and the peripheral blood of patients with advanced-stage B-NHL. The expression levels of miR-503 and KAI1 detected in B-NHL patients suggest that miR-503 may be involved in the occurrence and development of B-NHL through the regulation of KAI1 expression levels.

In conclusion, in the present study, the expression levels of miR-503 were found to be increased in the tissues and peripheral blood of B-NHL patients. However, the expression levels of KAI1 were decreased in B-NHL tissues and peripheral blood. Since miR-503 and KAI1 are associated with B-NHL, these findings may have a clinical significance in predicting recurrence and metastasis of B-NHL, based on miR-503 expression. Thus, miR-503 may have an application as a novel therapeutic and diagnostic marker in B-NHL patients.

\section{Acknowledgements}

This study was supported by a grant from the Natural Science Foundation of China (grant no. 81201793).

\section{References}

1. Nair VS, Pritchard CC, Tewari M and Ioannidis JP: Design and analysis for studying microRNAs in human disease: A primer on -omic technologies. Am J Epidemiol 180: 140-152, 2014.

2. Arora S, Swaminathan SK, Kirtane A, Srivastava SK, Bhardwaj A, Singh S, Panyam J and Singh AP: Synthesis, characterization and evaluation of poly (D,L-lactide-co-glycolide-based nanoformulation of miRNA-150: Potential implications for pancreatic cancer therapy. Int J Nanomedicine 9: 2933-2942, 2014.

3. Chen WX, Cai YQ, Lv MM, Chen L, Zhong SL, Ma TF, Zhao JH and Tang JH: Exosomes from docetaxel-resistant breast cancer cells alter chemosensitivity by delivering microRNAs. Tumour Biol 35: 9649-9659, 2014.

4. Guo J, Liu X and Wang M: miR-503 suppresses tumor cell proliferation and metastasis by directly targeting RNF31 in prostate cancer. Biochem Biophys Res Commun 64: 1302-3028, 2015.

5. Li B, Liu L, Li X and Wu L: miR-503 suppresses metastasis of hepatocellular carcinoma cell by targeting PRMT1. Biochem Biophys Res Commun 64: 982-987, 2015.

6. Xu YY, Wu HJ, Ma HD, Xu LP, Huo Y and Yin LR: MicroRNA-503 suppresses proliferation and cell-cycle progression of endometrioid endometrial cancer by negatively regulating cyclin D1. FEBS J 280: 3768-3779, 2013.

7. Lu YC, Chen YJ, Wang HM, Tsai CY, Chen WH, Huang YC, Fan KH, Tsai CN, Huang SF, Kang CJ, et al: Oncogenic function and early detection potential of miRNA-10b in oral cancer as identified by microRNA profiling. Cancer Prev Res (Phila) 5: 665-674, 2012.

8. Zhou J, Tao Y, Peng C, Gu P and Wang W: miR-503 regulates metastatic function through Rho guanine nucleotide exchanger factor 19 in hepatocellular carcinoma. J Surg Res 188: 129-136, 2014.

9. Xiao F, Zhang W, Chen L, Chen F, Xie H, Xing C, Yu X, Ding S, Chen K, Guo H, et al: MicroRNA-503 inhibits the G1/S transition by downregulating cyclin D3 and E2F3 in hepatocellular carcinoma. J Transl Med 11: 195, 2013. 
10. Fukui T, Fukumoto K, Okasaka T, Kawaguchi K, Nakamura S, Hakiri S, Ozeki N, Hirakawa A, Tateyama H and Yokoi K: Clinical evaluation of a new tumour-node-metastasis staging system for thymic malignancies proposed by the International Association for the Study of Lung Cancer Staging and Prognostic Factors Committee and the International Thymic Malignancy Interest Group. Eur J Cardiothorac Surg: Nov 7, 2015. (Epub ahead of print).

11. Yang Y, Liu L, Zhang Y, Guan H, Wu J, Zhu X, Yuan J and Li M: MiR-503 targets PI3K p85 and IKK- $\beta$ and suppresses progression of non-small cell lung cancer. Int J Cancer 135: 1531-1542, 2014

12. Zhou L, Wu SW, Yu L, Song WQ, Cheng ZN and Wang DN: The expression of KAI1 in gastric adenocarcinoma and relationship with angiogenesis/lymphangiogenesis. Sichuan Da Xue Xue Bao Yi Xue Ban 45: 43-48, 2014 (In Chinese).

13. Liu X, Guo XZ, Li HY, Chen J, Ren LN and Wu CY: KAI1 inhibits lymphangiogenesis and lymphatic metastasis of pancreatic cancer in vivo. Hepatobiliary Pancreat Dis Int 13: 87-92, 2014.

14. Xiu B, Lin Y, Grote DM, Ziesmer SC, Gustafson MP, Maas ML, Zhang Z, Dietz AB, Porrata LF, Novak AJ, et al: IL-10 induces the development of immunosuppressive CD14(+)HLA-DR(low/-) monocytes in B-cell non-Hodgkin lymphoma. Blood Cancer J 5: e328, 2015 .

15. Hua Q, Zhu Y and Liu H: Severe and fatal adverse events risk associated with rituximab addition to B-cell non-Hodgkin's lymphoma (B-NHL) chemotherapy: a meta-analysis.J Chemother. 2015 Apr 15: 1973947815Y0000000025. [Epub ahead of print].

16. Ghorbian S, Jahanzad I, Javadi GR and Sakhinia E: Evaluation diagnostic usefulness of immunoglobulin light chains $(\operatorname{Ig} \kappa, \operatorname{Ig} \lambda)$ and incomplete IGH D-J clonal gene rearrangements in patients with B-cell non-Hodgkin lymphomas using BIOMED-2 protocol. Clin Transl Oncol 16: 1006-1011, 2014.

17. Scwab M (Ed). Ann Arbor Staging System. In: Encyclopedia of Cancer. 2nd edition. Springer Berlin Heidelberg, Berlin, Heidelberg, pp 169-169, 2009.

18. Swerdlow SH, Campo E, Harris NL, et al (eds): WHO Classification of Tumours of Haematopoietic and Lymphoid Tissues. Vol 2. 4th edition. IARC Press, Lyon, France, 2008.

19. Yamashita Y, Hasegawa M, Deng Z, Maeda H, Kondo S, Kyuna A, Matayoshi S, Agena S, Uehara T, Kouzaki H, et al: Human papillomavirus infection and immunohistochemical expression of cell cycle proteins $\mathrm{pRb}, \mathrm{p} 53$, and p16(INK4a) in sinonasal diseases. Infect Agent Cancer 10: 23, 2015.

20. You J, Madigan MC, Rowe A, Sajinovic M, Russell PJ and Jackson P: An inverse relationship between KAI1 expression, invasive ability, and MMP-2 expression and activity in bladder cancer cell lines. Urol Oncol 30: 502-508, 2012.
21. Altman T, Travers M, Kothari A, Caspi R and Karp PD: A systematic comparison of the MetaCyc and KEGG pathway databases. BMC Bioinformatics 14: 112, 2013.

22. Sekimzu M, Mori T, Kikuchi A, Mitsui T, Sunami S, Kobayashi R, Fujita N, Inada H, Takimoto T, Saito AM et al: Prognostic impact of cytogenetic abnormalities in children and adolescents with mature B-cell non-Hodgkin lymphoma: A report from the Japanese Pediatric Leukemia/Lymphoma Study Group (JPLSG). Pediatr Blood Cancer 62: 1294-1296, 2015.

23. Bruni R, Marcantonio C, Pulsoni A, Tataseo P, De Angelis F, Spada E, Marcucci F, Panfilio S, Bianco P, Riminucci M, et al: microRNA levels in paraffin-embedded indolent B-cell nonHodgkin lymphoma tissues from patients chronically infected with hepatitis B or C virus. BMC Infect Dis 14 Suppl 5: S6, 2014.

24. Song A, Ye J, Zhang K, Yu H, Gao Y, Wang H, Sun L, Xing X, Yang $\mathrm{K}$ and Zhao M: Aberrant expression of the CHFR prophase checkpoint gene in human B-cell non-Hodgkin lymphoma. Leuk Res 39: 536-543, 2015

25. Wang K, Jiang Y,Zheng W, Liu Z, Li H, Lou J, Gu M, and Wang X: Silencing of Human Phosphatidylethanolamine-Binding Protein 4 Enhances Rituximab-Induced Death and Chemosensitization in B-Cell Lymphoma. PLoS One 8: e56829, 2013.

26. Di Lisio L, Sánchez-Beato M, Gómez-López G, Rodríguez ME, Montes-Moreno S, Mollejo M, Menárguez J, Martínez MA, Alves FJ, Pisano DG, et al: MicroRNA signatures in B-cell lymphomas. Blood Cancer J 2: e57, 2012

27. Tagawa H,Ikeda S and Sawada K: Role of microRNA in the pathogenesis of malignant lymphoma. Cancer Sci 104: 801-809, 2013.

28. Peng Y, Liu YM, Li LC, Wang LL and Wu XL: microRNA-503 inhibits gastric cancer cell grow th and epithelial-to-mesenchymal transition. Oncol Lett 7: 1233-1238, 2014.

29. Maurer CA, Graber HU, Friess H, Beyermann B, Willi D, Netzer P,Zimmermann A and Büchler MW: Reduced expression of the metastasis suppressor gene KAI1 in advanced colon cancer and its metastases. Surgery 126: 869-880, 1999.

30. Guo X, Friess H, Graber HU, Kashiwagi M, Zimmermann A, Korc M and Büchler MW: KAI1 expression is up-regulated in early pancreatic cancer and decreased in the presence of metastases. Cancer Res 56: 4876-4880, 1996

31. Yang JL, Jackson P, Yu Y, Russell PJ, Markovic B and Crowe PJ: Expression of the KAI1 metastasis suppressor gene in non-metastatic vs. metastatic human colorectal cancer. Anticancer Res 22. 3337-3342, 2002. 